\title{
EXPERIENCIAS DE LOS FAMILIARES CON PACIENTES EN LA UNIDAD DE TERAPIA INTENSIVA
}

THE RELATIVES' EXPERIENCES WITH PATIENTS IN THEINTENSIVE CARE UNIT

Recibido enero 2016 Aceptado agosto 2017

Correspondencia: Marcio Jiménez Gutiérrez Sección Veredas número 2 interior 401, Colonia Infonavit Delegación Iztacalco, C.P. 08900

Ciudad de México Tel. (s): 56492171 / 0445520865440 mail: el_megaov@hotmail.com

\section{Autores:}

Marcio Jiménez Gutiérrez

Especialista en Enfermería del Adulto en Estado Crítico, HRAE Ixtapaluca.

Viridiana Rojas Manzano

Especialista en Enfermería del Adulto en Estado Crítico, HRAE Ixtapaluca.

Ivonne Areli García Santa Olalla

Maestra en Salud Pública, HRAE Ixtapaluca.

Sonia González González

Maestra en Enfermería, HRAE Ixtapaluca.

Bertha Alicia Mancilla Salcedo

Maestra en Administración, HRAE Ixtapaluca.
Palabras clave: experiencias, expectativas, familia, cuidados intensivos, terapia intensiva.

Key words: experiences, expectative, family, critical care, intensive care. 


\section{RESUMEN}

Introducción: el ingreso de un usuario a unidades de cuidados criticos tiene repercusiones familiares que deben ser estudiadas para mejorar la atención proporcionada. El cuidado y las respuestas humanas son objeto de estudio del profesional de enfermería, sin embargo estas se han centrado de manera individual, dejando en término secundario a los familiares.

Objetivo: conocer las experiencias de familiares con pacientes en una unidad de cuidados intensivos adultos.

Metodología: estudio cualitativo, perspectiva fenomenológica, llevado a cabo en un hospital de tercer nivel. La población de estudio fueronfamiliares con pacientes hospitalizados en la Unidad de Terapia Intensiva Adultos; muestra por participantes voluntarios (muestra autoseleccionada de estudios cualitativos, el número de participantes se limitó hasta alcanzar la saturación teórica). Se empleó la entrevista semiestructurada y para el análisis de las mismas se usó el software MAXQDA (Qualitative Data Analysis Software for Mac and Windows).

Resultados: los familiares presentan cambios en la dinámica de vida como descuido personal, inestabilidad económica y cambios en la interacción social, expresan sentimientos negativos por el ingreso de su paciente a una unidad crítica y sus expectativas están relacionadas con la información, trato digno y atención eficaz.

Palabras clave: experiencias, expectativas, familia, cuidados intensivos, terapia intensiva.

\section{ABSTRACT}

Introduction: the patient's admission to the intensive care unit has consequences on their relatives that need to be studied to improve quality's attention. The care and human reiponses are objets of study of the nursing profesional, however they've been focused on the patient in an individual way leaving apart the relatives.

Objective: to know the experiences of patients relatives in the adult intensive care unit.

\section{Methodology: qualitative study,} phenomenological perspective, which was performed on a third level attention hospital. Study population were relatives with patients admitted in the Adult Intensive Care Unit; volunteer sample was chosen (auto - selected sample of qualitative studies, the number of participants were limited when data saturation was reached). We used the semi structured interview and for the analysis it was used the software MAXQDA (Qualitative Data Analysis Software for Mac and Windows).

Findings: the relatives show changes in dynamic of life as personal neglect, economic instability and social interaction changes, they show negative feelings because of the patient's admission to the intensive care unit and their expectations are related to information, dignified behavior and effective medical care.

Key words: experiences, expectative, family, critical care, intensive care. 


\section{INTRODUCCIÓN}

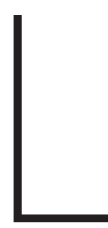

a Organización Mundial de la Salud señala que la enfermería abarca la atención autónoma y en colaboración, dispensada a personas de todas las edades, familias, grupos y comunidades, enfermos o no, y en toda circunstancia; de acuerdo con esta aseveración, podemos resaltar que la atención que se proporciona no solo debe estar dirigida hacia las personas desde el enfoque individual, sino familiar e incluso comunitario, sin importar el proceso salud - enfermedad en el que se encuentran o el nivel de atención donde se proporcionan las intervenciones.

Para dar cuidado al individuo, familia o comunidad, la enfermería requiere de un método específico que soporta su praxis al igual que otras disciplinas del área de la salud, dicho método es el Proceso de Enfermería (PE), que es el análogo del método científico simplificado, que se caracteriza por la sistematización lógica, la integración de toda experiencias y competencias: conocimientos, habilidades, actitudes y valores adquiridos por el o la enfermera para proporcionar cuidado a las respuestas humanas del paciente, con la calidad y calidez requeridas por la dignidad de la persona, ${ }^{2}$ este se encuentra estructurado por cinco etapas: valoración, diagnóstico, planeación, ejecución y evaluación, ${ }^{3}$ todas las etapas con interdependencia

Específicamente es la etapa diagnóstica del PE la que comprende el análisis de datos relacionados con la respuesta humana frente al proceso salud enfermedad ${ }^{4}$ con la finalidad de poder emitir un juicio clínico, es decir, un diagnóstico. De acuerdo con la NANDA (Asociación Norteamericana de Diagnósticos de Enfermería, por sus siglas en inglés), un diagnóstico de enfermería se define como un juicio clínico sobre las experiencias/respuestas de una persona, familia o comunidad frente a problemas de salud/procesos vitales reales o potenciales, ${ }^{5}$ resaltando a la familia como una de las unidades eje del juicio clínico de enfermería. Si bien los juicios clínicos enfermeros tienen el potencial de identificar necesidades en los grupos familiar y comunitario, sería importante señalar que las experiencias y respuestas humanas de estos últimos, dependiendo el espacio y nivel de atención, podrían ser desapercibidos e invisibilizados.

A nivel internacional, existen diversas investigaciones primarias y secundarias (artículos de revisión y revisiones sistemáticas) que se han interesado en las respuestas de la familia frente a la hospitalización de sus pacientes en unidades de cuidados críticos, concluyendo en la importancia de estudiar dichos procesos pues permiten mejorar la calidad del cuidado que debe ser proporcionado a la familia, y que en ocasiones suele ser ignorado o que pasa desapercibido por los profesionales de la salud. -11 $^{\text {-1 }}$ Dichos aspectos que ya han sido estudiados a nivel internacional contrastan con la producción científica del país. A nivel nacional existe escasa o nula evidencia cualitativa de los procesos vivenciales de la familia con pacientes hospitalizados en servicios críticos, lo que permite apertura a un tópico novel que puede ser estudiado y explotado en beneficio de los usuarios y familiares de los servicios de salud. Si bien existe vasta evidencia internacional, sería pertinente mencionar que dichos aspectos pueden variar dependiendo del espacio, cultura y contexto social donde sean estudiados, de ahí la importancia de reproducir investigaciones que den evidencia de la relevancia del tema en el entorno nacional.

El conocimiento de las experiencias de las familias del paciente crítico puede proporcionar herramientas para planificar estrategias de intervención para mejorar la calidad del cuidado a las familias con un paciente ingresado en UCl.

El fenómeno y la experiencia desde la perspectiva fenomenológica de Heidegger-Husserl busca la comprensión interpretativa del fenómeno, desvelar sentidos y significados de la vida cotidiana. La potencialidad de la investigación cualitativa puede generar e implementar proyectos que traigan valiosas contribuciones para la práctica de enfermería, por lo que la praxis enfermera no puede verse afectada de manera positiva únicamente mediante la metodología cuantitativa; mediante el enfoque fenomenológico el investigador está influido por relaciones de género, poder, ética, conocimiento,

\section{8} MMN Mhy $_{W}$ Enf Neurol Vol. 16. No. 1 enero - abril 2017 
técnicas y procedimientos científicos, el conocimiento no solo es objetivo y medible, puede ser sujeto a la interpretación y el significado del investigador y quienes participan en ella. ${ }^{12}$

El objetivo general de este estudio fue conocer las experiencias y expectativas de familiares con pacientes hospitalizados en una unidad de cuidados intensivos adultos.

\section{METODOLOGÍA}

巨 studio cualitativo, exploratorio, con perspectiva fenomenológica, que se llevó a cabo de mayo a diciembre del 2015 en un hospital de tercer nivel de la Secretaría de Salud. La población de estudio fueron los familiares con pacientes hospitalizados en la Unidad de Terapia Intensiva Adultos. Muestra por participantes voluntarios (muestra autoseleccionada de estudios cualitativos), los participantes respondieron de manera voluntaria a la invitación de los investigadores. El número de participantes se limitó a las personas entrevistadas durante el periodo de recolección de datos establecido en el cronograma y al alcanzar la saturación de categorías que es definida como la repetición o redundancia de los datos en donde los nuevos análisis confirman lo que se ha fundamentado. ${ }^{13}$

Los criterios de inclusión de las personas entrevistadas fueron familiares con pacientes hospitalizados en la Unidad de Terapia Intensiva Adultos de un hospital de tercer nivel en un periodo de tiempo de ingreso a la misma de las 24 a 72 horas, que aceptaron participar en el estudio y entrevista, con edad superior o igual a los 18 años de edad (mayoría de edad). Los criterios de exclusión a considerar fueron los familiares con pacientes hospitalizados en la Unidad de Terapia Intensiva Adultos que se encontraron ausentes y que no desearon participar en el estudio y entrevistas.

El estudio representó un riesgo mínimo para los familiares entrevistados y se apegó a los lineamientos de la Ley General de Salud y la Declaración de Helsinki. ${ }^{14}, 15$ La investigación tuvo aprobación por parte del comité de investigación del hospital (NR003-2015) y de ética respectivamente. Se respetó el anonimato, autonomía y privacidad de las personas participantes mediante el Consentimiento Informado, la accesibilidad a la información relacionada con la investigación, así como la libertad de participar, permanecer y desertar del estudio y entrevistas en cualquier momento.

Para la recolección de datos fue elaborado un guion de entrevista semiestructurada que incluyó introducción, desarrollo y conclusiones y que básicamente se encontraba centrada en tres preguntas eje que pretenden llegar al objetivo de la investigación (cuadro l). Las entrevistas fueron llevadas a cabo en un lugar cerrado, proporcionado y asignado por la Subdirección de Enfermería, que permitió y garantizó una adecuada interacción del entrevistado y entrevistador, siendo estas rotuladas y grabadas con equipo digital y formato mp3 para después ser transcritas para su análisis. La transcripción de las entrevistas fue elaborada a nivel literal y para verificar su precisión con la grabación digital dos de los investigadores corroboraron la misma de manera separada.

Para el análisis de datos, se empleó como herramienta el análisis de contenido (específicamente análisis del discurso)ycomosoporteparaelloseutilizó el programa MAXQDA (Qualitative Data Analysis Software for Mac and Windows). Se efectuó codificación en primer plano, análisis de cada unidad y posteriormente la elaboración de categorías.

\section{RESULTADOS}

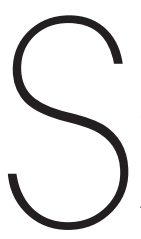
e presentan los datos arrojados de 19 entrevistas a familiares con las características ya mencionadas quienes compartieron sus experiencias. La información codificó en tres temas principales: Cambios en la dinámica de vida, Sentimientos y emociones y expectativas; Ios datos obtenidos de las entrevistas se agrupan en el siguiente cuadro (cuadro II). A continuación se ejemplifican estos con los aspectos más relevantes de las entrevistas. 


\section{Tema 1: Cambios en la dinámica de vida}

Son diversos los cambios en la dinámica de vida que experimentan los familiares desde el momento en que su paciente ingresa en una unidad crítica. Uno de los más significativos es el descuido personal y deficiencia en el autocuidado que experimentan al sobreponer sus necesidades frente a las de su paciente u originadas debido a la misma estancia intrahospitalaria:

me hicieron lo del azúcar, me había salido en 260, después anoche me la hicieron, estaba hasta los suelos la azúcar y la presión, no que... pues ya dos días sin comer como que de repente llega uno a su limite, ya tu cuerpo no resiste, y estar aqui día y noche... (F17).

Otros familiares señalan que el cambio en la dinámica de vida más importante está relacionado con la inestabilidad económica asociada con la pérdida de empleo y la agudización de la precariedad económica, y quetambién ocasionada por los costos de atención hacia los pacientes:

he dejado mi trabajo (...) y este y también que pues lo que me vayan pidiendo que necesite eso o lo otro, pues de dónde lo voy a comprar si no estoy trabajando, no estoy generando, eh... el dinero, el poco dinero que traía pues ya me lo estoy acabando, y después, después cómo le voy a hacer... (F5).

Otros familiares entrevistados relatan que existen cambios en la interacción familiar y social debido al tiempo que se necesita del familiar dentro del hospital para información o el consentimiento sobre procedimientos a realizar (transfusiones y cirugías):

mis hijos, me desaparté de ellos, porque no puedo estar aquí, con mi esposo y con los niños, es muy desesperante estar así porque uno quisiera estar, dividirse en dos partes para estar en los dos lados y no se puede, es muy difícil vivir todo esto... (F13).

Asimismo, se relata por diversos familiares el desgaste físico que impacta en su vida y que es generado por la estancia de su paciente en una unidad crítica: pos este, pos se junta todo, cansancio, que no (...) aunque sí yo siento que ando pisando en las nubes, vaya, no estoy, siento que no... pues hasta que no salga yo creo que voy a estar... bien... (F3).

\section{Tema 2: Sentimientos y Emociones}

Además de los cambios en la dinámica de vida, los familiares experimentan emociones y sentimientos negativos que son expresados de la siguiente manera:

Nostalgia: ... se vuelve uno como más sensible, no sé si influya el ver al ser humano entubado, con un ventilador atrás, lo haga uno valorar más la vida (...) Cuando tienes un paciente en terapia intensiva, recuerdas todo, todo, todo, ya sea del paciente, en primer lugar, y también la trayectoria en la vida en combinación con el paciente. (F6)

Ira: ...en ocasiones nosotros por la preocupación de nuestro paciente nos ponemos un poco exaltados, o eso, o a veces hasta groseros, ¿no? y este, y, sí, pues hay algunos que, que tienen la delicadeza de soportarnos y de controlarnos... (F5).

Tristeza: ... mucha tristeza de verla a ella así. Y este.. con ese accidente que tuvo ella, pues es... me parece muy triste verla a ella así. De momento estaba bien y de momento la veo como ahorita, pues sí me siento triste. (F16).

Incertidumbre: ... el estar viendo que se apaga su lucecita de vida, aún y que los médicos pues luego le digan a uno que probablemente esté saliendo adelante, pero esa incertidumbre que vive uno de estar aquí, día y noche, esperando que en cualquier momento le digan a uno esa lucecita ya se apagó, es una experiencia muy fuerte y muy dura... (F74).

Angustia: Mi vida es una vida totalmente desesperante y angustiante por el motivo en el que se encuentra mi esposo. (F2).

Dolor: una presión así en el pecho, o sea, como un dolor interno, o sea, de verlo en la situación que está, este, pues es doloroso verlo en la situación que está, este, no, nunca hubiera querido verlo así este, porque, pues lo quiero mucho... (F5). 
Impotencia: quisiera uno... o sea, ayudarlo, poderle... o sea, al verlo como qué gestos hace, de que está sufriendo, quisiera uno... darle un algo, que le quite el dolor, o se siente uno impotente de no poderlo ayudar, no sé, mil cosas que a la vez no te lo sé explicar, mmm... muy difícil todo esto. (F13).

\section{Tema 3: Expectativas}

Si bien el ingreso de un usuario en una unidad crítica es un suceso impactante para los familiares, a partir de este surgen diversas expectativas y necesidades que los profesionales de la salud pueden ser capaces de solventar. Cabe resaltar que dichas expectativas se encuentran dentro de un marco de posibilidades donde los familiares tienen conocimiento de los límites de la atención que proporciona el personal de salud en enfermedades complejas que requieren de estancia en unidades críticas:

Yo entiendo que hay veces que se hace todo, o sea, ellos le brindan toda la atención a nuestros pacientes y eso, pero a veces no está en sus manos... (F5).

Por lo que una de las principales expectativas de los familiares (que en diversas ocasiones es verbalizada en forma de agradecimiento), distante de la solución de lasv afecciones por las que su paciente ingresó a la terapia intensiva, es la de información adecuada:

Me explicó los riesgos que puede tener mi esposo y eso pues me parece bien porque este me platica o me dice las cosas muy adecuadamente. (F2).

dudas que tengo pues se las pregunto a las enfermeras, a los doctores, dándome ellos buenas respuestas, me sientan, me platican... (F12).

Dicha expectativa no solamente está relacionada con el informe médico, sino que puede surgir en el tiempo de visita del familiar y solventada por otros profesionales de la salud como es el caso de enfermería:

no sé, tengo la costumbre de ver, ipor qué está sonando así señorita este aparato? Ah, porque por esto, o como ahorita que me tocó ver que lo está inyectando, oiga, disculpe, ¿qué le está inyectando?
No le estoy inyectando, nada más es solución porque se le tapó el suero (...) me han contestado muy bien a lo que yo les pregunto, me han explicado muy bien hasta eso, bien, todo muy bien. (F13).

Si bien la información puede ser adecuada, existen exigencias sobre la manera en cómo debe ser proporcionada; al respecto uno de los familiares señala lo siguiente:

Y luego aquí hay doctores que no te dan ninguna esperanza, van de lleno a los madrazos, perdón que te lo diga, son secos, ¿sí? No hay sensibilidad de alguna manera, nada, entonces, esto está así y su señora va a quedar... quién sabe si despierte, quién sabe si esto, no te dan este... solamente un doctor que ha sido un poco más... más humano yo creo, sí, pero pues si la realidad es tremenda pues deben de, deben ser más sensibles ellos, ¿no? (F7).

Otras de las expectativas son aquellas relacionadas con una adecuada atención sanitaria, tanto en atención eficaz como en trato digno que en diversas entrevistas también es mencionado como felicitación o en forma de agradecimiento:

Mi mamá, o sea, al principio, sí tuvo un buen concepto del servicio que dan todos, todos, todos, todos. Atención, que es lo primordial, la atención y todo... (F9).

a mí el personal, sea de trabajo social, los médicos, las enfermeras, he tenido, esta es la segunda experiencia que tengo en el sector salud de la secretaría de salubridad y asistencia. Y me parece buena, me parece buena... (F6).

La verdad, honestamente le voy a decir, jqué finos, eh! Ha sido una atención muy, muy buena (...) La verdad honestamente yo nunca pensé que se iban a tener las atenciones que se han tenido, el personal con una calidad humana como no tiene una idea. (Fl4).

Otra de las expectativas fue el deseo sobre la prolongación del tiempo de visita:

a veces también lo mejor es que quisiéramos estar un ratito más con ellos, que es muy poquito el rato que pasamos con ellos, es lo que pienso... (F4). 
Diversos familiares señalaron que la flexibilidad en trámites administrativos $y$ en la normatividad es un área de oportunidad factible de mejorar por los profesionales de la salud:

por ejemplo si yo voy a pedir otro informe me mandan a otro lado (...) pues de allá me mandaron para acá y aquí me dicen que vaya para allá y la policía me dice que me espere... (F3).

.. nos dijo que estaba prohibido tener cobijas aquí, que si queríamos podíamos tenerlas allá abajo y estar ahí abajo, pero pues si nuestro paciente está aquí arriba y uno tiene que estar aquí... ahora, es una noche, quieras o no una noche es pesada, y si nos vence el sueño pues de perdida una cobija para taparnos, nada más eso, es lo único que pedimos. (F79).

\section{DISCUSIÓN Y CONCLUSIONES}

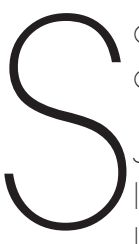

on diversas las investigaciones que han abordado los aspectos mencionados.

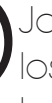
Johansson-Rnanicrnt estudia a profundidad os sentimientos y respuestas emocionales de los padres al ingreso de su hijo en una unidad crítica; los resultados de su estudio muestran como el sentimiento incertidumbre está relacionada con cuestiones sobre la supervivencia y posibles secuelas de discapacidad y coinciden con once de los familiares entrevistados en nuestro estudio. A-Mutair y colaboradores realizaron una revisión sobre las necesidades de los familiares en unidades críticas quienes señalan la importancia de los estudios cualitativos sobre el tema y las limitaciones de su revisión al carecer esta de estudios cuantitativos en su mayoría, el estudio presente es una fortaleza frente a este panorama. Obringer y colaboradores realizaron un estudio cuantitativo sobre las necesidades de los familiares en unidades de cuidados intensivos, el cual concluye que las principales necesidades están relacionadas con la de información honesta, ${ }^{8}$ y que tiene relación con nuestro estudio pues once de las personas entrevistadas indicaron la importancia de la misma en forma de agradecimiento o demanda.
El estudio de Frizon y colaboradores concluye que los principales sentimientos expresados por los familiares en unidades críticas son la angustia, impotencia y tristeza ${ }^{10}$ que muestra similitud con este trabajo pues son los sentimientos que conforman parte de la experiencia de los familiares.

Otros aspectos visibilizados y de importancia son aquellos relacionados con el descuido personal y deficiencia de autocuidado que expresan algunos familiares, ocasionado por el ingreso de su paciente en una unidad crítica al sobreponer el tiempo de estancia que se requiere en salas de espera para autorización de trámites y consentimientos sobre sus necesidades básicas o relacionadas con atención en salud que ellos requieren también. La inestabilidad económica que expresan los familiares es otro de los aspectos mencionados derivados de la estancia intrahospitalaria de su paciente y los costos de la atención. Por último es importante señalar que el sentimiento de incertidumbre es uno de los más mencionados en la experiencia de los familiares y que podrían tener relación con las necesidades de información y atención eficaz que también pueden ser solventadas por el personal de salud.

Consideramos que los resultados de este trabajo son aspectos a considerar por parte del personal de salud en la atención del enfermo crítico. Dichos aspectos pueden representar áreas de oportunidad en los familiares de estos pacientes y pueden favorecer la calidad del cuidado multidisciplinario que se otorga.

\section{Reconocimientos:}

Los autores deseamos agradecer a la Red de Unidades de Investigación en Enfermería ENEO, Instituciones e Institutos Nacionales de Salud y a la Lic. María Eugenia Francisca López de Silanes por el apoyo otorgado para realizar este trabajo. Agradecimientos especiales al Dr, Rodolfo Pinto Almazán y el Lic. Daniel de Jesús Rosales Mendoza por el apoyo para la aprobación del proyecto y el soporte en la búsqueda de información respectivamente.

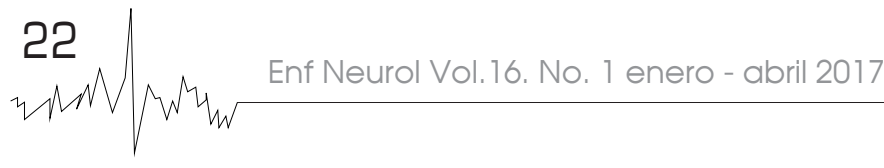


Cuadro I. Preguntas centrales del formato de entrevista semiestructurada. ¿De qué manera ha cambiado su vida al tener un paciente en la Unidad de Terapia Intensiva? ¿Qué sentimientos han surgido desde que ingresó su paciente a la Unidad de Terapia Intensiva? ¿Qué expectativas tiene del personal de salud hacia su paciente y hacia usted?

Cuadro II. Datos obtenidos de las entrevistas semiestructuradas.

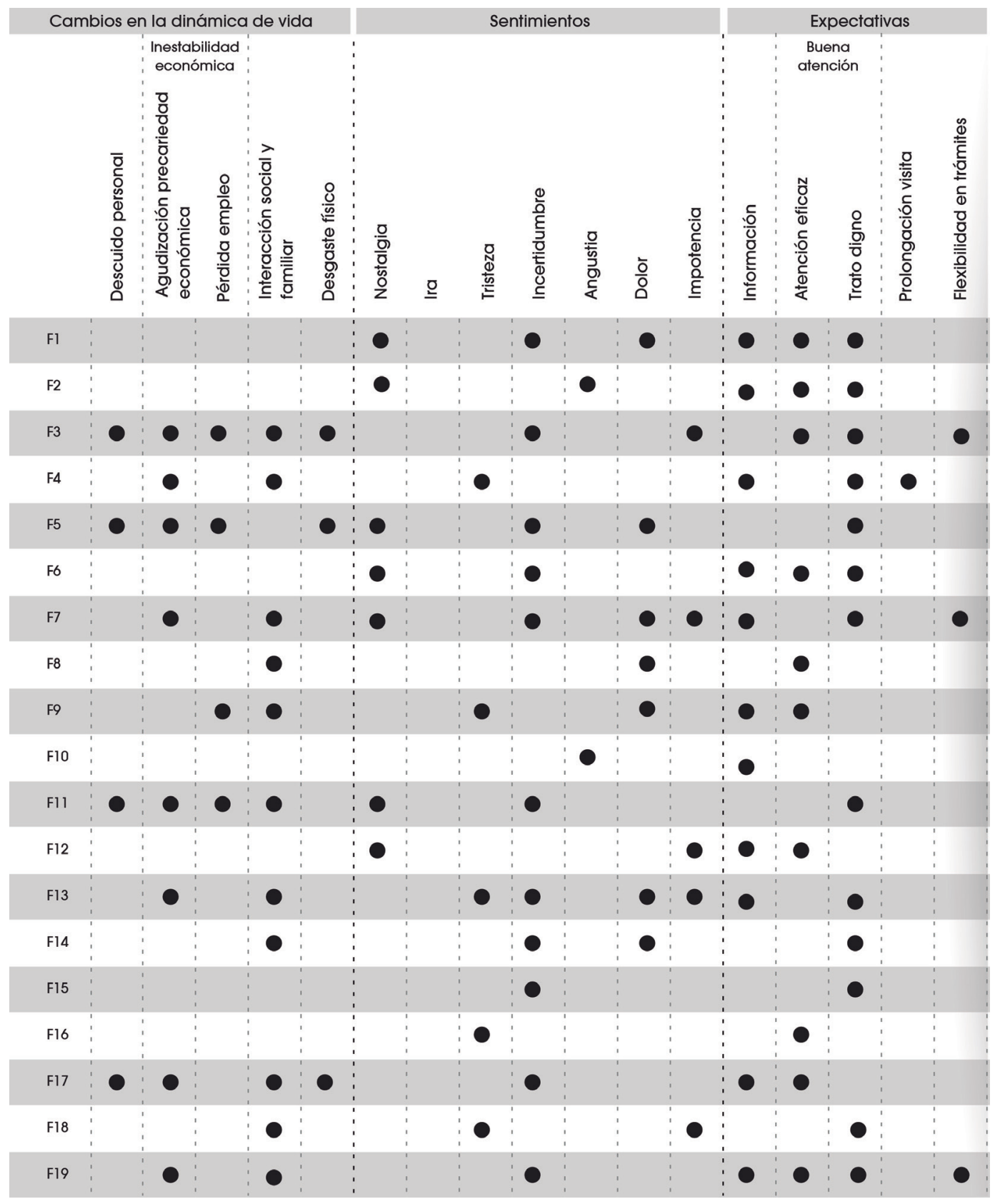




\section{REFERENCIAS BIBLIOGRÁFICAS}

1. Organización Mundial de la Salud. Temas de Salud. Enfermería. Disponible en: URL: hHtp://www.who.int/topics/nursing/es/

2. Rodríguez-Sánchez BA. Proceso enfermero. $2^{\underline{a}}$ ed. México: Ediciones Cuellar; 2003

3. Secretaría de Salud. NOM-019-SSA3-2013, Para la práctica de enfermería en el Sistema Nacional de Salud. Disponible en: URL: http://dof.gob.mx/ nota_detalle.php?codigo=5312523\&fecha=02/09/2013

4. Morán-Aguilar V. Mendoza-Robles AL. Marco de referencia. En: Aguilar V. Mendoza-Robles AL. Proceso de enfermería uso de lenguajes NANDA, NIC y NOC modelos referenciales. $3^{a}$ ed. México: Trillas; 2010. p. 75

5. Herdman TH. Diagnósticos enfermeros: definiciones y clasificación 20122014. España: Elsevier; 2012

6. Johansson-Rnanicrnt I. Emotional responses of family members of a critically ill patient: a hermeneutic analysis. IJEMHHR 2014;16(3):5-8,

7. Al-Mutair AS, Plummer V. O'Brien A, Clerehan R. Family needs and involvement in the intensive care unit: a literature review. Journal of Clinical Nursing 2013;22:1805-1817

8. Obringer K, Hilgenberg C, Booker K. Needs of adult family members of intensive care unit patients. Journal of Clinical Nursing 2012;21:1651-1658.
9. Pardavila-Belio MI, Vivar CG. Necesidades de la familia en las unidades de cuidados intensivos. Revisión de la literatura. Enferm Intensiva 2012:23(2):51-67.

10. Frizon G, Nascimiento ERP, Bertoncello KCG, Martins JJ. Familiares na sala de espera de uma unidade de terapia intensiva; sentimientos revelados Rev Gaúcha Enferm 2011:32(1):72-78.

11. Verhaeghe S, Defloor T, Van-Zuuren F, Duiinstee M, Grypdonck M. The needs and experiences of family members of adult patients in an intensive care unit: a review of the literature. Journal of Clinical Nursing 2004;74:507-509

12. Rocha-Moreira RC, Mendonca-Lopez RL, Andrade-Santos N. Entrevisto fenomenológica: peculiaridades para la producción científica de enfermería. Index Enferm:2013:22(1).

13. Hernández-Sampieri R, Fernández-Collado C, Baptista-Lucio MP Metodología de la investigación. 5ำed. Perú: Mc Graw Hill; 2010.

14. Reglamento de la Ley General de Investigación en materia de Investigación para la Salud. Disponible en: URL: http://www.salud.gob.mx/ unidades/cdi/nom/compi/rlgsmis.html

15. Declaración de Helsinki de la asociación médica mundial. Principios éticos para las investigaciones médicas en seres humanos. Disponible en URL: http://www.wma.net/es/30publications/10policies/b3/17c_es.pdf

\section{4}

